Journal of Universal Mathematics

Vol.3 No.1 PP.94-102 (2020)

ISSN-2618-5660

\title{
ORDERING METHODS OF C-CONTROL CHARTS WITH INTERVAL TYPE-2 INTUITIONISTIC FUZZY SETS
}

\author{
S.DEMIRALP AND G. HACAT
}

\begin{abstract}
The control charts are important because it provides information about the process and the state of control of the product. With this feature, control charts are used in many areas. Intuitionistic fuzzy sets and intervalvalued intuitionistic fuzzy sets are useful to modelling of the real-world problems. Ordering of the fuzzy numbers is investigated by a lot of scientists and it is generalised to intuitionistic fuzzy sets because of applicability. Ordering interval-valued type-2 intuitionistic fuzzy sets is significant in making decision, data analysis and artificial intelligence since interval analysis is required.In this study a new method to ordering interval-valued type-2 intuitionistic fuzzy sets is defined and numerical methods are compared to other methods.
\end{abstract}

\section{INTRODUCTION}

Zadeh introduced Fuzzy set theory in 1965[8]. Later on Atanassov generalized the concept of fuzzy set and introduced the idea of Intuitionistic fuzzy set $[2,3,5]$. The concept of fuzzy numbers and arithmetic operations with these numbers were first introduced and investigated by Chang and Zadeh and others. There exist a large amount of literature involving the ranking of fuzzy numbers and decision making with fuzzy numbers $[1,6]$. The notions of intuitionistic fuzzy numbers in different context were studied in and applied in multi criteria decision making problems. Intuitionistic fuzzy sets (IFSs), characterized by three functions expressing degrees of membership, non-membership, and hesitation (or indeterminacy), have received increasing attention since their introduction by Atanassov.

Atanassov and Gargov [4] proposed the notion of the intervalvalued intuitionistic fuzzy set (IVIFS), which is characterized by a membership function and a nonmembership function whose values are intervals rather than exact numbers. Based on IVIFS, Xu [9] defined the notion of interval-valued intuitionistic fuzzy number (IVIFN) and introduced some operations on IVIFNs. IVIFNs have huge amount of application in decision making processes.

Recently few methods for ranking IFNs has also been introduced Wang and Zhang defined the trapezoidal Intuitionistic fuzzy number (TRIFN) and gave ranking method which transformed the ranking of TRIFNs in to ranking of interval numbers. Other than Wang and Zhang, Mitchell[10] introduced ranking of IFNs. Nayagam et al.[11] described Ifns of special type and introduced a method of IF

Key words and phrases. Interval type-2 intuitionistic fuzzy sets,Fuzzy control charts,c-Control charts,ordering methods. 
scoring of IFNs as a generalization of Chen and Hwang's scoring for ranking IFNs. In many applications, ranking of interval-valued intuitionistic fuzzy numbers is an important component of the decision-raking process.

Control charts are important for process or product because they provide information about the control situation of process and product. Because of this feature, control charts are used in many fields. Information about the product and/or process, which is under control or not, can be provided by looking the control charts. Fuzzy numbers are used to reduce information losses in operations with crisp numbers. In control charts applications the fuzzy set theory reduces the information losses and provide more flexible decision-making process.

The purpose of the study is to create an innovation using the ranking methods, which has not used for control charts in accessible literature, for the fuzzy control charts with interval type- 2 intuitionistic fuzzy sets.In this paper attention has been paid to the study of a new ranking procedure for trapezoidal intuitionistic fuzzy number (TRIFN). There are numerous methods for ranking of simple fuzzy numbers but, we lack of effective methods for ranking of intuitionistic fuzzy numbers (IFN). To demonstrated our proposed approach one numerical example has been presented.

\section{Type-2 Intuitionistic Fuzzy Set}

We start this section by introducing some basic concepts related to intervalvalued intuitionistic trapezoidal fuzzy numbers, which will be used throughout this paper.

Definition 2.1. A generalized fuzzy set called intuitionistic fuzzy sets, shown as follows:

$$
A=\left\{\left\langle x, \mu_{A}(x), \nu_{A}(x)\right\rangle: x \in X\right\} .
$$

In which, $\mu_{A}$ means a membership function, and $\nu_{A}$ means a non-membership, with the condition $0 \leq \mu_{A}(x)+\nu_{A}(x) \leq 1, \mu_{A}(x), \nu_{A}(x) \in[0,1]$ for all $x \in X$.

Definition 2.2. An interval-valued intuitionistic fuzzy set in $A$ over $X$ is an object having the form:

$$
A=\left\{\left\langle x, \widetilde{\mu}_{A}(x), \widetilde{\nu}_{A}(x)\right\rangle: x \in X\right\},
$$

$0 \leq \sup \widetilde{\mu}_{A}(x)+\sup \nu_{A}(x) \leq 1, \forall x \in X$.

The intervals $\widetilde{\mu}_{A}(x)$ and $\widetilde{\nu}_{A}(x)$ denote, respectively, the membership function and the non-membership function of the element $x$ to the set $A$. Thus for each $x \in$ $X, \widetilde{\mu}_{A}(x)$ and $\widetilde{\nu}_{A}(x)$ are closed intervals and their lower and upper end points are, respectively, denoted by $\widetilde{\mu}_{A L}(x), \widetilde{\mu}_{A U}(x), \widetilde{\nu}_{A L}(x)$ and $\widetilde{\nu}_{A U}(x)$. To compare IVIFNs, $\mathrm{Xu}$ and Chen (2007) introduced the score function and the accurate function as follows:

Definition 2.3. (IVIFNs Score Function). Let $\widetilde{\alpha}=\left(\left[\mu^{-}, \mu^{+}\right],\left[\nu^{-}, \nu^{+}\right]\right)$be an interval-valued intuitionistic fuzzy number, a score function $S_{X}(\widetilde{\alpha})$ of an intervalvalued intuitionistic fuzzy value can be represented as follows

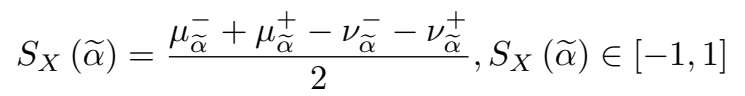

to evaluate the degree of score of the interval-valued intuitionistic fuzzy value $\widetilde{\alpha}=$ $\left(\left[\mu^{-}, \mu^{+}\right],\left[\nu^{-}, \nu^{+}\right]\right)$, where $S_{X}(\widetilde{\alpha}) \in[-1,1]$. The lager the value of $S_{X}(\widetilde{\alpha})$, the more the degree of score of the interval-valued intuitionistic fuzzy number $\widetilde{\alpha}$. 
Definition 2.4. (IVIFNs Accuracy Function). Let $\widetilde{\alpha}=\left(\left[\mu^{-}, \mu^{+}\right],\left[\nu^{-}, \nu^{+}\right]\right)$be an interval-valued intuitionistic fuzzy number, an accuracy function $H_{X}(\widetilde{\alpha})$ of an interval-valued intuitionistic fuzzy value can be represented as follows

$$
H_{X}(\widetilde{\alpha})=\frac{\mu_{\widetilde{\alpha}}^{-}+\mu_{\widetilde{\alpha}}^{+}+\nu_{\widetilde{\alpha}}^{-}+\nu_{\widetilde{\alpha}}^{+}}{2}, H_{X}(\widetilde{\alpha}) \in[0,1]
$$

to evaluate the degree of accuracy of the interval-valued intuitionistic fuzzy value $\widetilde{\alpha}=\left(\left[\mu^{-}, \mu^{+}\right],\left[\nu^{-}, \nu^{+}\right]\right)$, where $H_{X}(\widetilde{\alpha}) \in[0,1]$. The lager the value of $H_{X}(\widetilde{\alpha})$, the more the degree of accuracy of the interval-valued intuitionistic fuzzy number $\widetilde{\alpha}$.

Definition 2.5. (IVIFNs Novel Accuracy Function). Let $\widetilde{\alpha}=\left(\left[\mu^{-}, \mu^{+}\right],\left[\nu^{-}, \nu^{+}\right]\right)$ be an interval-valued intuitionistic fuzzy number, a novel accuracy function $M_{X}(\widetilde{\alpha})$ of an interval-valued intuitionistic fuzzy value can be represented as follows

$$
M_{X}(\widetilde{\alpha})=\mu^{-}+\mu^{+}-1+\left(\nu^{-}+\nu^{+}\right) / 2, M_{X}(\widetilde{\alpha}) \in[-1,1]
$$

to evaluate the degree of novel accuracy of the interval-valued intuitionistic fuzzy value $\widetilde{\alpha}=\left(\left[\mu^{-}, \mu^{+}\right],\left[\nu^{-}, \nu^{+}\right]\right)$, where $M_{X}(\widetilde{\alpha}) \in[-1,1]$. The lager the value of $M_{X}(\widetilde{\alpha})$, the more the degree of novel accuracy of the interval-valued intuitionistic fuzzy number $\widetilde{\alpha}$.

However, the domains of IFSs and IVIFSs are discrete sets, and therefore they are only used to indicate the extent to which the criterion does or does not belong to some fuzzy concepts. For example, their membership degrees and non-membership degrees are only relative to a fuzzy concept "Excellent" or "Good". To overcome this flaw, Wan (2011) developed the interval-valued intuitionistic trapezoidal fuzzy set (IVITFS), which extends discrete set to continuous set and is the generalization of IFSs and IVIFSs.

Definition 2.6. (IVITFS). Let $\widetilde{\alpha}$ is an interval-valued intuitionistic trapezoidal fuzzy set (IVITFS), its interval-valued membership function is:

$$
\mu_{\widetilde{\alpha}}(x)=\left\{\begin{array}{c}
\frac{x-a}{b-a} \mu_{\widetilde{\alpha}}, a \leq x<b \\
\mu_{\widetilde{\widetilde{\alpha}}}, b \leq x \leq c \\
\frac{d-x}{d-c} \mu_{\widetilde{\alpha}}, c<x \leq d \\
0, \text { others }
\end{array}\right.
$$

and

$$
\mu_{\widetilde{\alpha}}^{+}(x)=\left\{\begin{array}{c}
\frac{x-a}{b-a} \mu_{\widetilde{\alpha}}^{+}, a \leq x<b \\
\mu_{\widetilde{\widetilde{\alpha}}}^{+}, b \leq x \leq c \\
\frac{d-x}{d-c} \mu_{\widetilde{\alpha}}^{+}, c<x \leq d \\
0, \text { others }
\end{array}\right.
$$

its interval-valued non-membership function is:

$$
\nu_{\widetilde{\alpha}}^{-}(x)=\left\{\begin{array}{c}
\frac{b-x+\nu_{\widetilde{\alpha}}^{-}(x-a)}{b-a}, a \leq x<b \\
\nu_{\widetilde{\alpha}}^{-}, b \leq x \leq c \\
\frac{x-c+\nu_{\widetilde{\alpha}}(d-x)}{d-c}, c<x \leq d ; \\
1, \text { others }
\end{array}\right.
$$


and

$$
\nu_{\widetilde{\alpha}}^{+}(x)=\left\{\begin{array}{c}
\frac{b-x+\nu_{\tilde{\alpha}}^{+}(x-a)}{b-a}, a \leq x<b ; \\
\nu_{\widetilde{\alpha}}^{+}, b \leq x \leq c ; \\
\frac{x-c+\nu_{\tilde{\alpha}}^{+}(d-x)}{d-c}, c<x \leq d ; \\
1, \text { others } .
\end{array}\right.
$$

where $0 \leq \mu_{\widetilde{\alpha}}^{-} \leq \mu_{\widetilde{\alpha}}^{+} \leq 1 ; 0 \leq \nu_{\widetilde{\alpha}}^{-} \leq \nu_{\widetilde{\alpha}}^{+} \leq 1 ; 0 \leq \mu_{\widetilde{\alpha}}^{+}+\nu_{\widetilde{\alpha}}^{+} \leq 1 ; a, b, c, d \in R$. Then

$$
\widetilde{\alpha}=\left([a, b, c, d] ;\left[\mu_{\widetilde{\alpha}}^{-}, \mu_{\widetilde{\alpha}}^{+}\right] ;\left[\nu_{\widetilde{\alpha}}^{-}, \nu_{\widetilde{\alpha}}^{+}\right]\right)
$$

is called an interval-valued intuitionistic trapezoidal fuzzy set(IVITFS), which is shown in Fig. 1. To compare the definition of interval-valued intuitionistic trapezoidal fuzzy set with other fuzzy sets, we introduce the definition of trapezoidal interval type-2 fuzzy set (Chen and Lee,2010). A trapezoidal interval type-2 fuzzy set can be described as follows:

$$
\begin{aligned}
\widetilde{\widetilde{A}} & =\left(\widetilde{A}^{U}, \widetilde{A}^{L}\right) \\
& =\left(\left(a^{U}, b^{U}, c^{U}, d^{U} ; H_{1}\left(\widetilde{A}^{U}\right), H_{2}\left(\widetilde{A}^{U}\right)\right),\left(a^{L}, b^{L}, c^{L}, d^{L} ; H_{1}\left(\widetilde{A}^{L}\right), H_{2}\left(\widetilde{A}^{L}\right)\right)\right)
\end{aligned}
$$

where $\widetilde{A}^{U}$ and $\widetilde{A}^{L}$ denote the upper and lower membership functions of the interval type- 2 fuzzy set $\widetilde{\widetilde{A}}$,respectively. The trapezoidal interval type-2 fuzzy set is shown in Fig.2.

To understand these definitions of fuzzy sets, we make a comparison as follows:

\section{itbpF2.5659in1.5402in0inFigure}

Fig.1 The interval-valued intuitionistic trapezoidal

fuzzy set

\section{itbpF2.693in1.6501in0inFigure}

Fig.2 The trapezoidal interval type-2 fuzzy set

(1) If $a^{U}=a^{L}=a, b^{U}=b^{L}=b, c^{U}=c^{L}=c$ and $d^{U}=d^{L}=d$, then $\widetilde{\widetilde{A}}=$ $\left(a, b, c, d ;\left[H_{1}(\widetilde{A}), H_{2}(\widetilde{A})\right]\right)$, which is reduced to the interval-valued intuitionistic trapezoidal fuzzy set $\widetilde{\alpha}$. Therefore, the interval-valued intuitionistic trapezoidal fuzzy set $\widetilde{\alpha}$ is a special case of trapezoidal interval type-2 fuzzy set $\widetilde{\widetilde{A}}$.

(2) The interval-valued intuitionistic trapezoidal fuzzy set is a generalization of IFSs and IVIFSs, The IFSs and IVIFSs have some limitation in expressing comprehensive decision variables because that their domains are discrete sets. Different from the concept of IFSs and IVIFSs, the membership degrees and nonmembership degrees of IVITFS are not relative to a fuzzy concept "Excellent" or "Good", but relative to the trapezoidal fuzzy number. Thus, an IVITFS $\widetilde{\alpha}=$ $\left([a, b, c, d] ;\left[\mu_{\widetilde{\alpha}}, \mu_{\widetilde{\alpha}}^{+}\right] ;\left[\nu_{\widetilde{\alpha}}^{-}, \nu_{\widetilde{\alpha}}^{+}\right]\right)$can express an well-known quantity "approximate $\mathrm{x}$ ", which is expressed using any value between a and $\mathrm{d}$ with different degrees of membership and degrees of non-membership. For example, let

$$
\widetilde{6}=([4,5,6,7] ;[0.5,0.7] ;[0.1,0.2])
$$

be an IVITFN, then, when $x=5$, its membership degree to be $\widetilde{6}$ is $[0.5,0.7]$, its nonmembership degree to be $\widetilde{6}$ is $[0.1,0.4]$. Obviously, the domain of IVITFN is a consecutive set, and therefore IVITFNs are a generalization of IVIFNs. 
(3) If $\left[\mu_{\widetilde{\alpha}}^{\bar{\alpha}}, \mu_{\widetilde{\alpha}}^{+}\right]=[1,1]$ and $\left[\nu_{\widetilde{\alpha}}^{-}, \nu_{\widetilde{\alpha}}^{+}\right]=[0,0]$, then the IVITFN $\widetilde{\alpha}$ degenerates to $\alpha=([a, b, c, d] ; 1 ; 0)$, which is just about a trapezoidal fuzzy number (TFN).Therefore the concept of the IVITFN is generalization of that of TFN. Furthermore, if $b=c, \mu_{\widetilde{\alpha}}^{-}=\mu_{\widetilde{\alpha}}^{+}$and $\nu_{\widetilde{\alpha}}^{-}=\nu_{\widetilde{\alpha}}^{+}$, then $\widetilde{\alpha}$ reduces to a ITFN (Shuet al., 2006). The additional parameters $\left[\mu_{\widetilde{\alpha}}^{-}, \mu_{\widetilde{\alpha}}^{+}\right]$and $\left[\nu_{\widetilde{\alpha}}^{-}, \nu_{\widetilde{\alpha}}^{+}\right]$are introduced to reflect the confidence level and non-confidence level of the IVITFN $\widetilde{\alpha}$, respectively.

The arithmetical operations over IVITFNs may be defined as follows.

Definition 2.7. (Operation Law of IVITFS). Let

and

$$
\widetilde{\alpha}_{1}=\left(\left[a_{1}, b_{1}, c_{1}, d_{1}\right] ;\left[\mu_{\widetilde{\alpha}_{1}}^{-}, \mu_{\widetilde{\alpha}_{1}}^{+}\right] ;\left[\nu_{\widetilde{\alpha}_{1}}^{-}, \nu_{\widetilde{\alpha}_{1}}^{+}\right]\right)
$$

$$
\widetilde{\alpha}_{2}=\left(\left[a_{2}, b_{2}, c_{2}, d_{2}\right] ;\left[\mu_{\widetilde{\alpha}_{2}}^{-}, \mu_{\widetilde{\alpha}_{2}}^{+}\right] ;\left[\nu_{\widetilde{\alpha}_{2}}^{-}, \nu_{\widetilde{\alpha}_{2}}^{+}\right]\right)
$$

be two interval-valued intuitionistic trapezoidal fuzzy numbers, and $\lambda \geq 0$, then

(1) $\widetilde{\alpha}_{1} \oplus \widetilde{\alpha}_{2}=\left(\left[a_{1}+a_{2}, b_{1}+b_{2}, c_{1}+c_{2}, d_{1}+d_{2}\right]\right.$;

$$
\begin{array}{r}
\left.\left[\mu_{\widetilde{\alpha}_{1}}^{-}+\mu_{\widetilde{\alpha}_{2}}^{-}-\mu_{\widetilde{\alpha}_{1}}^{-} \mu_{\widetilde{\alpha}_{2}}^{-}, \mu_{\widetilde{\alpha}_{1}}^{+}+\mu_{\widetilde{\alpha}_{2}}^{+}-\mu_{\widetilde{\alpha}_{1}}^{+} \mu_{\widetilde{\alpha}_{2}}^{+}\right],\left[\nu_{\widetilde{\alpha}_{1}}^{-} \nu_{\widetilde{\alpha}_{2}}^{-}, \nu_{\widetilde{\alpha}_{1}}^{+} \nu_{\widetilde{\alpha}_{2}}^{+}\right]\right) \\
=\left(\left[a_{1}+a_{2}, b_{1}+b_{2}, c_{1}+c_{2}, d_{1}+d_{2}\right] ; \min \left[\mu_{\widetilde{\alpha}_{1}}, \mu_{\widetilde{\alpha}_{2}}\right] ; \max \left[v_{\widetilde{\alpha}_{1}}, v_{\widetilde{\alpha}_{2}}\right]\right)
\end{array}
$$

(2) $\widetilde{\alpha}_{1} \otimes \widetilde{\alpha}_{2}=\left(\left[a_{1} a_{2}, b_{1} b_{2}, c_{1} c_{2}, d_{1} d_{2}\right]\right.$;

$$
\begin{gathered}
\left.\left[\mu_{\widetilde{\alpha}_{1}}^{-} \mu_{\widetilde{\alpha}_{2}}^{-}, \mu_{\widetilde{\alpha}_{1}}^{+} \mu_{\widetilde{\alpha}_{2}}^{+}\right],\left[\nu_{\widetilde{\alpha}_{1}}^{-}+\nu_{\widetilde{\alpha}_{2}}^{-}-\nu_{\widetilde{\alpha}_{1}}^{-} \nu_{\widetilde{\alpha}_{2}}^{-}, \nu_{\widetilde{\alpha}_{1}}^{+}+\nu_{\widetilde{\alpha}_{2}}^{+}-\nu_{\widetilde{\alpha}_{1}}^{+} \nu_{\widetilde{\alpha}_{2}}^{+}\right]\right) \\
=\left(\left[a_{1} a_{2}, b_{1} b_{2}, c_{1} c_{2}, d_{1} d_{2}\right] ; \min \left[\mu_{\left.\left.\widetilde{\alpha}_{1}, \mu_{\widetilde{\alpha}_{2}}\right] ; \max \left[v_{\widetilde{\alpha}_{1}}, v_{\widetilde{\alpha}_{2}}\right]\right)}\right.\right.
\end{gathered}
$$

(3) $\lambda \widetilde{\alpha}=\left([\lambda a, \lambda b, \lambda c, \lambda d] ;\left[1-\left(1-\mu_{\widetilde{\alpha}}\right)^{\lambda}, 1-\left(1-\mu_{\widetilde{\alpha}}^{+}\right)^{\lambda}\right],\left[\left(\nu_{\widetilde{\alpha}}^{-}\right)^{\lambda},\left(\nu_{\widetilde{\alpha}}^{+}\right)^{\lambda}\right]\right)$

(4) $\widetilde{\alpha}^{\lambda}=\left(\left[a^{\lambda}, b^{\lambda}, c^{\lambda}, d^{\lambda}\right] ;\left[\left(\mu_{\widetilde{\alpha}}^{-}\right)^{\lambda},\left(\mu_{\widetilde{\alpha}}^{+}\right)^{\lambda}\right],\left[1-\left(1-v_{\widetilde{\alpha}}^{-}\right)^{\lambda}, 1-\left(1-v_{\widetilde{\alpha}}^{+}\right)^{\lambda}\right]\right)$.

A useful tool for dealing with FSs is their $\alpha$-cuts. Every $\alpha$-cut of a fuzzy number is a closed interval and a family of such intervals describes completely a fuzzy number under study. Recall that IVIFS are particular cases of IVITFNs. Then, IVITFNs can have the same score funtion $S_{X}(\widetilde{\alpha})$ and accurate funtion $H_{X}(\widetilde{\alpha})$ as IVIFS.

Definition 2.8. A $\alpha$-cut set of a IVITFN $\widetilde{\alpha}=\left([a, b, c, d] ;\left[\mu_{\widetilde{\alpha}}^{-}, \mu_{\widetilde{\alpha}}^{+}\right] ;\left[\nu_{\widetilde{\alpha}}^{-}, \nu_{\widetilde{\alpha}}^{+}\right]\right)$is a crisp subset of $R$ defined as

$$
\widetilde{\alpha}_{\alpha}=\left\{x: \mu_{\widetilde{\alpha}}(x) \geq \alpha\right\}
$$

where $0 \leq \alpha \leq \mu_{\widetilde{\alpha}}$.

Definition 2.9. A $\beta$-cut set of a IVITFN $\widetilde{\alpha}=\left([a, b, c, d] ;\left[\mu_{\widetilde{\alpha}}^{-}, \mu_{\widetilde{\alpha}}^{+}\right] ;\left[\nu_{\widetilde{\alpha}}^{-}, \nu_{\widetilde{\alpha}}^{+}\right]\right)$is a crisp subset of $R$ defined as

$$
\widetilde{\alpha}_{\beta}=\left\{x: v_{\widetilde{\alpha}}(x) \leq \beta\right\}
$$

where $v_{\widetilde{\alpha}} \leq \beta \leq 1$.

It can be easily followed from the definition of IVITFN, Definition 2.8 and Definition 2.9 that $\widetilde{\alpha}_{\alpha}$ and $\widetilde{\alpha}_{\beta}$ are both closed sets and are denoted by $\widetilde{\alpha}_{\alpha}=$ $\left[L_{\widetilde{\alpha}}(\alpha), R_{\widetilde{\alpha}}(\alpha)\right]$ and $\widetilde{\alpha}_{\beta}=\left[L_{\widetilde{\alpha}}(\beta), R_{\widetilde{\alpha}}(\beta)\right]$ respectively. The respective values of $\widetilde{\alpha}_{\alpha}$ and $\widetilde{\alpha}_{\beta}$ are calculated as follows:

$$
\begin{aligned}
& {\left[L_{\widetilde{\alpha}}(\alpha), R_{\widetilde{\alpha}}(\alpha)\right]=\left[a+\frac{\alpha(b-a)}{\mu_{\widetilde{\alpha}}}, d-\frac{\alpha(d-c)}{\mu_{\widetilde{\alpha}}}\right]} \\
& {\left[L_{\widetilde{\alpha}}(\beta), R_{\widetilde{\alpha}}(\beta)\right]=\left[\frac{(1-\beta) b+\left(\beta-v_{\widetilde{\alpha}}\right) a}{\mu_{\widetilde{\alpha}}}, \frac{(1-\beta) c+\left(\beta-v_{\widetilde{\alpha}}\right) d}{\mu_{\widetilde{\alpha}}}\right] .}
\end{aligned}
$$


Definition 2.10. The support of a IVITFN $\widetilde{\alpha}=\left([a, b, c, d] ;\left[\mu_{\widetilde{\alpha}}^{-}, \mu_{\widetilde{\alpha}}^{+}\right] ;\left[\nu_{\widetilde{\alpha}}^{-}, \nu_{\widetilde{\alpha}}^{+}\right]\right)$ for the membership function is defined as

$$
\sup p_{\mu}(\widetilde{\alpha})=\left\{x: \mu_{\widetilde{\alpha}}(x) \geq 0\right\} .
$$

Definition 2.11. The support of a IVITFN $\widetilde{\alpha}=\left([a, b, c, d] ;\left[\mu_{\widetilde{\alpha}}^{-}, \mu_{\widetilde{\alpha}}^{+}\right] ;\left[\nu_{\widetilde{\alpha}}^{-}, \nu_{\widetilde{\alpha}}^{+}\right]\right)$ for the non-membership function is defined as

$$
\sup p_{\nu}(\widetilde{\alpha})=\left\{x: v_{\widetilde{\alpha}}(x) \leq 1\right\} .
$$

\section{Ranking Methods for Interval Type-2 Intuitionistic Fuzzy Sets}

In this section, two ranking methods are used; Chen et al.'s ranking method and Qin and Liu's ranking method,respectively.

3.1. Chen et al.'s ranking method. Chen et al. proposed ranking method for interval type-2 trapezoidal intuitionistic fuzzy sets. Ranking of $A$ is shown as follow:

$$
\begin{aligned}
R V\left(\widetilde{\widetilde{A}_{i}}\right)= & {\left[\frac{\left(a_{i}+K_{i}\right)+\left(d_{i}+K_{i}\right)+\left(H_{1}\left(\widetilde{A_{i}}\right)+H_{2}\left(\widetilde{A_{i}}\right)\right)}{2}\right] } \\
& *\left[\frac{\left(a_{i}+K_{i}\right)+\left(b_{i}+K_{i}\right)+\left(c_{i}+K_{i}\right)+\left(d_{i}+K_{i}\right)}{4}\right]
\end{aligned}
$$

$K_{i}$ value is the values that makes the numbers positive, the $K_{i}$ value will be evaluated as 0 ,since there will be no negative data for the control charts, in this study.

3.2. Qin and Liu's ranking method. Qin and Liu proposed ranking method for type-2 intuitionstic fuzzy sets. Ranking of $A$ is shown as follow:

$$
\begin{aligned}
\operatorname{Rank}(A)= & M_{1}(A)+M_{2}(A)+M_{3}(A)-\frac{1}{4}\left(S_{1}(A)+S_{2}(A)+S_{3}(A)+S_{4}(A)\right) \\
& +H_{1}(A)+H_{2}(A) .
\end{aligned}
$$

\section{C-Control Charts with Interval Type-2 Intuitionistic Fuzzy Sets}

Linguistic data can be represented by fuzzy sets. For this reason, there are lots of applications in many areas. Control charts that can be regarded as one of these fields. It is suitable for control charts, especially attribute control charts, because of the data are linguistic and categorical.

The attribute control charts are separated by the fraction rejected as nonconforming to the specifications, number of nonconforming items, number of nonconformities and number of nonconformities per unit. In this study, we have been working on the fuzzifing of control charts dealing with the number of nonconformities referred to as c control charts. For classical c control charts, control limit are calculated as given below.

$$
\begin{aligned}
C L & =\bar{c} \\
L C L & =\bar{c}-3 \sqrt{\bar{c}} \\
U C L & =\bar{c}+3 \sqrt{\bar{c}}
\end{aligned}
$$

where $\bar{c}$ is the mean of the nonconformities.

In this study,each sample point is expressed as a interval type- 2 trapezoidal intuitionistic fuzzy numbers $\left(a, b, c, d ;\left[H_{1}(\widetilde{A}), H_{2}(\widetilde{A})\right]\right)$. 
The fuzzy control limits are then calculated using the operations of interval type- 2 trapezoidal intuitionistic fuzzy sets. These equations are shown as follow:

$$
\begin{aligned}
\widetilde{C L} & =\left([\bar{a}, \bar{b}, \bar{c}, \bar{d}] ; \min \left[\mu_{\widetilde{\alpha}}, \mu_{\widetilde{\alpha}}^{+}\right] ; \max \left[\nu_{\widetilde{\alpha}}^{-}, \nu_{\widetilde{\alpha}}^{+}\right]\right) \\
& =\left(\left[\frac{\sum_{i=1}^{m} a_{i}}{m}, \frac{\sum_{i=1}^{m} b_{i}}{m}, \frac{\sum_{i=1}^{m} c_{i}}{m}, \frac{\sum_{i=1}^{m} d_{i}}{m}\right] ; \min \left[\mu_{\widetilde{\alpha}}^{-}, \mu_{\widetilde{\alpha}}^{+}\right] ; \max \left[\nu_{\widetilde{\alpha}}^{-}, \nu_{\widetilde{\alpha}}^{+}\right]\right) \\
\widetilde{U C L} & =\left((\bar{a}+3 \sqrt{\bar{a}}, \bar{b}+3 \sqrt{\bar{b}}, \bar{c}+3 \sqrt{\bar{c}}, \bar{d}+3 \sqrt{\bar{d}}) ; \min \left[\mu_{\widetilde{\alpha}}^{-}, \mu_{\widetilde{\alpha}}^{+}\right] ; \max \left[\nu_{\widetilde{\alpha}}^{-}, \nu_{\widetilde{\alpha}}^{+}\right]\right) \\
\widetilde{L C L} & =\left((\bar{a}-3 \sqrt{\bar{d}}, \bar{b}-3 \sqrt{\bar{c}}, \bar{c}-3 \sqrt{\bar{b}}, \bar{d}-3 \sqrt{\bar{a}}) ; \min \left[\mu_{\widetilde{\alpha}}, \mu_{\widetilde{\alpha}}^{+}\right] ; \max \left[\nu_{\widetilde{\alpha}}^{-}, \nu_{\widetilde{\alpha}}^{+}\right]\right)
\end{aligned}
$$

After calculating interval type-2 control limits, the ranking methods mentioned in the previous section are used to compare limits based on the data.

\section{Numerical Example}

In this section, numerical example is given so that the methods can be better understood. Data for nonconformities are shown in Table 1, which shows crisp value of data, and Table 2, which shows linguistic values of data. Interval type-2 trapezoidal fuzzy sets are transformed from linguistic data and control limits are obtained as interval type-2 trapezoidal numbers using Eqs. (4.4)-(4.6).

$\widetilde{C L}, \widetilde{U C L}$ and $\widetilde{L C L}$ are calculated as interval type- 2 trapezoidal fuzzy sets and these are given below,

$$
\begin{aligned}
\widetilde{C L} & =(18.13,22.67,26.93,32.07 ;[0.63,0.59] ;[0.48,0.45]) \\
& =(18.13,22.67,26.93,32.07 ;[0.48] ;[0.59]) \\
\widetilde{U C L} & =(30.88,36.95,42.47,49.05 ;[0.63,0.59] ;[0.48,0.45]) \\
& =(30.88,36.95,42.47,49.05 ;[0.48] ;[0.59]) \\
\widetilde{L C L} & =(1.15,7.13,12.65,19.32 ;[0.63,0.59] ;[0.48,0.45]) \\
& =(1.15,7.13,12.65,19.32 ;[0.48] ;[0.59])
\end{aligned}
$$

Table 1. Crisp values for numerical example itbpF4.337in1.2445in0inFigure

Table 2. Linguistic values for numerical example itbpF3.4402in2.0392in0inFigure

Table 3 shows ranking values using Chen et al.'s ranking method for numerical example.

Table 3. Ranking values using Chen et al.'s method itbpF2.3454in2.3289in0inFigure

Based on the calculations, the control chart is drawn using Chen et al.'s method in Figure 2.

$$
\text { itbpF3.5008in2.3929in0inFigure }
$$

Figure 2. Control charts with Chen et al.'s method 
Table 4 shows ranking values using Qin and Liu's ranking method for numerical example.

Table 4. Ranking values using Qin and Liu's method itbpF2.5218in2.6333in0inFigure

The control chart is depicted using Qin and Liu's method in Figure 3.

itbpF3.9704in2.6809in0inFigure

Figure 3. Control charts with Qin and Liu's method

When looking Fig. 4, the sample points of 3, 4, 7, 8, 11, 14-17 and 30 are out of control, while the others are in control.

\section{itbpF3.0882in2.0825in0inFigure}

Figure 4. Control charts with classical method

The last stage of study is the comparison of methods with each other. Table 5 is about comparison of all methods.

Table 5. Comparisons of classical control chart with ranking methods.

itbpF4.4711in3.0502in0inFigure

Conclusions

In the study, firstly, interval type 2 intuitionistic fuzzy control limits are set. After that, control charts are created with two different ranking methods. Then, the results obtained from ranking methods' are compared with the classical c-control chart, and the consistency of the results are investigated.

An important contribution of this study is that not only the control limits are calculated as interval type-2 intuitionistic fuzzy sets but also the ranking values. The other important point of this study is that it is the first study that tests ranking methods for fuzzy control charts. For further studies, this research can be extended to include effects of different ranking methods for interval type-2 intuitionistic fuzzy sets.

\section{REFERENCES}

[1] C.H. Cheng, "A new approach for ranking fuzzy numbers by distance method". Fuzzy Sets and Systems 95, 307-317, 1998.

[2] K. Atanassov, Intuitionistic sets, VII ITKR's Session,Sofia,June,1983.

[3] K. Atanassov, Intuitionistic fuzzy sets, Fuzzy sets and sytems 20,1986.

[4] K. Atanassov and G.Gargov,Interval-valued intuitionistic fuzzy sets, Fuzzy Sets and Sytems $31(3), 343-349,1989$.

[5] K. Atanassov, Operators over interval-valued intuitionistic fuzzy sets, Fuzzy Sets and Sytems 64(2),159-174,1994.

[6] S. Chen, "Ranking fuzzy numbers with maximizing set and minimizing set". Fuzzy Sets and Systems, 17,113-129,1985.

[7] S.Senturk and J.Antucheviciene,Interval type-2 fuzzy c-control charts:An application in a food company.Informatica,28(2),269-283,2017.

[8] L. A. Zadeh, "Fuzzy sets". Information and Control, 8(3),338-356, 1965.

[9] Z. S.Xu, "Intuitionistic fuzzy aggregation operators"IEEE Transactions on Fuzzy Systems,15(6),1179-1187, 2007. 
[10] H. B. Mitchell, "Ranking intuitionistic fuzzy numbers".International Journal of Uncertainty, Fuzziness and Knowledge Based Systems, 12(3), 377-386, 2004.

[11] L. Nayagam, V. Venkateshwari, and G. Sivaraman, "Ranking of intuitionistic fuzzy numbers".In Proceedings of the IEEE international conference on fuzzy systems (IEEE FUZZ 2008), 1971-1974.

(S.Demiralp) Kastamonu University, Mathematics Department, 37000, Kastamonu, Turkey Current address: Kastamonu University, Mathematics Department, 37000, Kastamonu, Turkey

E-mail address, G. Hacat: sdemiralp@kastamonu.edu.tr

(G. Hacat) Kastamonu University, Mathematics Department, 37000, Kastamonu, Turkey E-mail address, author two: ghacat@ogr.kastamonu.edu.tr 